\title{
Drying of a single droplet to investigate process-structure- function relationships: a review
}

\author{
Céline Sadek • Pierre Schuck • Yannick Fallourd • \\ Nicolas Pradeau • Cécile Le Floch-Fouéré • \\ Romain Jeantet
}

Received: 22 April 2014 / Revised: 23 July 2014 / Accepted: 18 August 2014

(C) INRA and Springer-Verlag France 2014

\begin{abstract}
Drying usually results in various types of particle morphology that influence the properties and the functionality of powders. Understanding how the final shape of the particle is formed is therefore a key issue for industrial applications. However, it is difficult to carry out relevant studies and interpret them on an industrial scale, mainly because of the complexity and scale of the drying operation and the difficulty of monitoring drying parameters in situ. To overcome these difficulties, experiments are currently performed with the simplest system conceivable: drying of a single droplet in a controlled environment. Different approaches are available in the literature in order to study the drying of a single droplet, from a sessile droplet to a free-flying droplet. This review aims to illustrate results obtained with these experiments, and their key contributions to fundamental and applied understanding of drying (or evaporation) phenomena are discussed. It is shown that investigation of a single droplet may be a key approach to spray drying in order to improve understanding of the chemical and physical mechanisms involved in particle processing and governing the properties of the final particle.
\end{abstract}

Keywords Spray drying · Particle formation · Powder · Droplet evaporation · Functionality

\footnotetext{
This paper is part of the special issue dedicated to the 10th anniversary of the INRA-Agrocampus Ouest joint research unit.

C. Sadek $\cdot$ Y. Fallourd $\cdot$ N. Pradeau

United Pharmaceuticals, 75000 Paris, France

C. Sadek · P. Schuck - C. Le Floch-Fouéré · R. Jeantet

INRA, UMR1253, 35000 Rennes, France

C. Sadek $\cdot$ P. Schuck $\cdot$ C. Le Floch-Fouéré $\cdot$ R. Jeantet $(\square)$

Agrocampus Ouest, UMR1253, 35000 Rennes, France

e-mail: romain.jeantet@agrocampus-ouest.fr
} 


\section{Introduction}

Spray drying is technology which has been widely used over the past decades in the manufacturing of food, biochemical, and pharmaceutical products. Its expansion has been due to the possibility of running such operations on a large scale, their ease of operation, and the ability to produce composite materials (Chow et al. 2007). It consists of spraying of the feed solution into a cloud of droplets within a flow of hot air. The water is rapidly evaporated from the droplets, resulting in dried particles. Despite the fact that it is a widely used technique, the drying process involves several fundamental and simultaneous transfer (heat, mass, and momentum), flow, phase transition, and mechanical instability. Moreover, drying of a wide range of polydisperse droplets flying in a hot air stream makes it difficult to investigate these mechanisms (Adhikari et al. 2000). The fast-drying kinetics (order of magnitude of $1 \mathrm{~s}$ ) clearly complicates the sampling and the recording of physical phenomena taking place in each droplet during intermediate stages of drying (Fu et al. 2012). Studies are difficult to carry out on an industrial scale and to interpret because of the complexity and scale of the drying equipment, the cost of experimental trials, and the difficulty of monitoring the various processing parameters in situ.

To overcome these difficulties, experiments are currently performed with the simplest system conceivable: drying of a single droplet in a controlled environment. Experiments with single droplets represent an easier way to observe and record the progressive drying of a droplet through kinetics indices with the recording of evolution of mass, temperature and diameter, rheological changes, skin formation, and physical deformations. This experimental concept represents a complementary approach to spray drying in order to improve understanding of fundamental phenomena governing drying that is not yet accessible on an industrial scale. This paper provides an overview of the different single droplet results and their contributions to fundamental and applied information on drying phenomena. The aim here is to provide key elements to improve understanding of the chemical and physical mechanisms involved in particle processing and that govern the properties of the final particle. The paper is organized as follows: Section 2 describes experimental techniques, Section 3 summarizes theoretical aspects of evaporation, Section 4 identifies results concerning particle formation, and Section 5 reports various factors related to particle properties. The last section describes different applications requiring particle engineering.

\section{Experimental techniques}

Ranz and Marshall (1952) were the first to investigate the drying of single suspended droplets of pure and dissolved component solutions (Ranz and Marshall 1952). The aim of their experiments was to study heat and mass transfer from a cloud of droplets to the surrounding air during spray drying. This simple approach was subsequently extended to other key issues in the spray drying process such as skin formation on a droplet and control of both the morphology and functionality of particles. The study of a single droplet has been considerably improved since the conception of new devices that allow scientists to record the drying phenomenon in situ and on a microscale. The following sections provide a brief overview of the main single-droplet experiments according to their historical evolution: levitated droplets, free-flying droplets, and sessile droplets (Fig. 1). It 
should be noted that a single droplet evolves in a fixed environment with controlled temperature, air velocity, and humidity, whatever the experiment.

\subsection{Levitated droplet}

This method investigating the drying of a single droplet by levitation, i.e., by applying an opposite force to gravity forces in order to keep a droplet suspended in the air, can be achieved through (i) an intrusive method involving a droplet suspended from a solid support or (ii) a non-intrusive method involving a free droplet lifted by physical forces. These two methods were the first single-droplet experiments and are well described in several papers (Adhikari et al. 2000; Fu et al. 2012; Schutyser et al. 2012; Vehring 2008).

In contact levitation experiments, a droplet of $1-\mathrm{mm}$ diameter is suspended from the tip of a thin glass filament or a glass capillary tube and placed in a controlled air stream (Fig. 1a). The drying parameters, such as the evolution of diameter, temperature, and mass of the droplet, are monitored by a camera and by thermocouples (one inserted in the core of the droplet and the other in the chamber), and the mass loss is recorded by a microbalance connected to the glass filament or by translating the glass filament deflection to the loss of droplet mass using an established calibration curve. Thermocouples allow the recording of the difference of temperature between the chamber and the droplet in real time. Due to its ease of use, the latter setup proposed by Ranz and Marshall (1952) has frequently been used in drying research to investigate the drying kinetics and particle morphology of a wide range of solutions (milk, eggs, organic and inorganic substances) (Eslamian and Ashgriz 2007; Lin and Gentry 2003; Lin and Chen 2002; Walton and Mumford 1999b). On the other hand, in the non-contact levitation method, the single droplet is kept in the air by means of acoustic or aerodynamic fields (Fig. 1b). The principle of acoustic levitation was described in detail by Davis et al. (1981): a standing wave is provided by an ultrasound generator in a levitation chamber where the evolution of a millimeter droplet is followed by an infrared thermometer and by a camera to record temperature and morphology data, respectively (Davis et al. 1981; Miura et al. 1977; Schiffter and Lee 2007). In the aerodynamic levitation method, the droplet freely floated on top of a vertical gas stream, used in combination with $\mathrm{CO}_{2}$ laser as a heating system (Hennet et al. 2011; Sakai et al. 2005).

\subsection{Free-flying droplet}

The second technique consists of generating a single or a chain of uniform free-falling droplets in a controlled air stream at the top of a drying tower, the temperature and air flow of which are recorded by thermo-sensors (Fig. 1c). This experimental setup is


Fig. 1 Experimental techniques used to study the drying of a single droplet

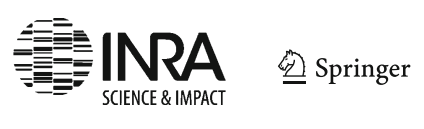


therefore closer to spray drying conditions. Droplets of micrometer range are generated by means of a micro-syringe or a pulsed orifice of identical volume and trajectory inside the chamber. Each droplet thus experiences the same drying history, resulting in particles of homogeneous shape and size. First described by Alexander and King (1985) and Elsayed et al. (1990), this experimental setup has considerably improved over the last decade (Elsayed et al. 1990). Wu et al. developed a generator of monodispersed droplets based on a piezoceramic system (Wu et al. 2007). In this device, the solution is placed in a reservoir and pressurized through a glass nozzle with a $50-\mu \mathrm{m}$ diameter orifice, and a fixed frequency is applied to the piezoceramic system to induce Rayleigh-like instability, followed by periodic breakup of the liquid jet in order to form a well-defined droplet chain. This method has made possible significant investigations into how process parameters impact on the quality of a powder, such as the physical properties of particles, their morphology, and their functionality (rehydration and encapsulation properties) (Fang et al. 2012; Rogers et al. 2012; Vehring et al. 2007).

\subsection{Sessile droplet}

This technique consists of gently depositing a droplet (nanometer to millimeter size) on a hydrophobic surface placed inside a dry well where the environmental conditions are controlled (Fig. 1d). The hydrophobic surface is required to maintain the spherical shape of the droplet, and this is achieved by specific treatment of the surface or by specific surface topography (Xu and Choi 2012; Yu et al. 2012). Evaporation of a droplet deposited on a hydrophobic substrate was studied by Mangel and Baer (1962), and this method was then frequently used to investigate thermodynamic flux inside a droplet and the mechanical properties of the particle skin (Hampton et al. 2012; Mangel and Baer 1962; Manukyan et al. 2013; Marín et al. 2012; McHale et al. 2005; Pauchard and Couder 2004). Most of these setups involved optical instruments (electronic and photonic) in order to image the evolution of the droplet shape during evaporation and to confirm data from mass records by a microbalance. Although this method has been established since 1962, and it is of considerable interest for nanotechnology applications, the analogy between the drying behavior of a sessile droplet and what can happen in a spray drier has only recently been proposed (Eslamian and Ashgriz 20077; Perdana et al. 2013; Wu et al. 2014). For example, Perdana et al. (2013) have developed a hightechnology setup including pneumatic micro-dispenser, drying chamber with air flow system, and camera system that is as close as possible to the conditions in a spray drying chamber. The sessile droplet configuration has also been used to investigate the spatial evolution of components inside the droplet and the physical mechanisms involved in skin formation using very sensitive instruments such as small-angle neutron scattering (Bahadur et al. 2011), small-angle x-ray scattering (Chen et al. 2012), or high sensitivity digital camera (Marín et al. 2011).

\subsection{Limitations}

Single-droplet experiments have been criticized as being unrealistic regarding spray drying techniques because the experimental droplets are larger and their drying behavior can be influenced by the experimental drying device, their static state, and the experimental environment. Heat and mass transfer can be affected by heat conduction 
between the droplet and the solid support (filament or hydrophobic support) or even by fluctuation in the acoustic field. Heating of levitated droplet by radiation from laser beams is quite different to spray drying where heat transfer is provided by convection (Adhikari et al. 2000). Moreover, it is also as difficult to monitor the changes in mass, temperature, and shape of droplets inside a monodispersed drying tower as in a spray drying tower. Despite these drawbacks, the drying of a single droplet under controlled conditions is still a valuable method to obtain real-time information regarding the basic mechanisms occurring such as transport mechanisms (Hampton et al. 2012), shrinkage behavior of droplets (Fu et al. 2013), skin formation (Parisse and Allain 1997), and buckling phenomena (Tsapis et al. 2005). Therefore, this approach has been applied to a large range of substances and solvents (Walton and Mumford 1999a). Therefore, in the following parts, the review will try to give a realist overview of these interesting outcomes obtained using these single-droplet drying setups.

\section{Theoretical aspects}

Frohn and Roth well defined the evaporation as a mass and heat flow process dependent for a single droplet with a radius $R_{s}$ and a temperature $T$ put into an environment with a temperature $T_{\infty}$ and the mass fraction $c_{\infty}$ of the vapor of the droplet material (Frohn and Roth 2000). In other words, evaporation can be treated as a matter of interdependent transfer of matter, heat, and movement flows which can be transferred through three different mechanisms as follows: diffusion/conduction, radiation, and convection (Fig. 2). Diffusion and conduction may occur through contact between substances in a static system. The radiation mechanism results from the propagation of electromagnetic waves, and convection represents transfer by up and down movements of gas and liquids. In the case of the evaporation process, which is considered to be a simultaneous heat and mass transfer operation, heat is transferred by conduction and convection from hot air to the liquid surface and converted to latent heat, whereas surface water molecules are transferred by diffusion and convection back into the air.

Studying evaporation of a droplet of water deposited on a solid surface or moving in air is a more complex problem. The process is non-stationary; the system is exposed to temperature, vapor, and surface tension gradients involving convection inside and all around the water droplet. Moreover, in view of the substrate, heat transfer by conduction into the substrate, and convection transfer induced by surface tension and temperature gradients between the substrate and droplet may also need to be considered.

\subsection{Evaporation of a single and pure liquid droplet}

The diffusion-controlled approach is commonly used to explain the evaporation process for both a model case and for more complex situations (Cabazat and Guéna 2010; Erbil 2012; Frohn and Roth 2000; Kim and Cho 2006). This approach is based on the analogy between water diffusion rate and heat conduction rate during the evaporation process.

When the liquid-vapor interface is in thermodynamic equilibrium, the rate of droplet evaporation is predicted to be the same as the rate of mass diffusion at any given moment. Taking all previous assumptions into consideration, Maxwell expressed Fick's 


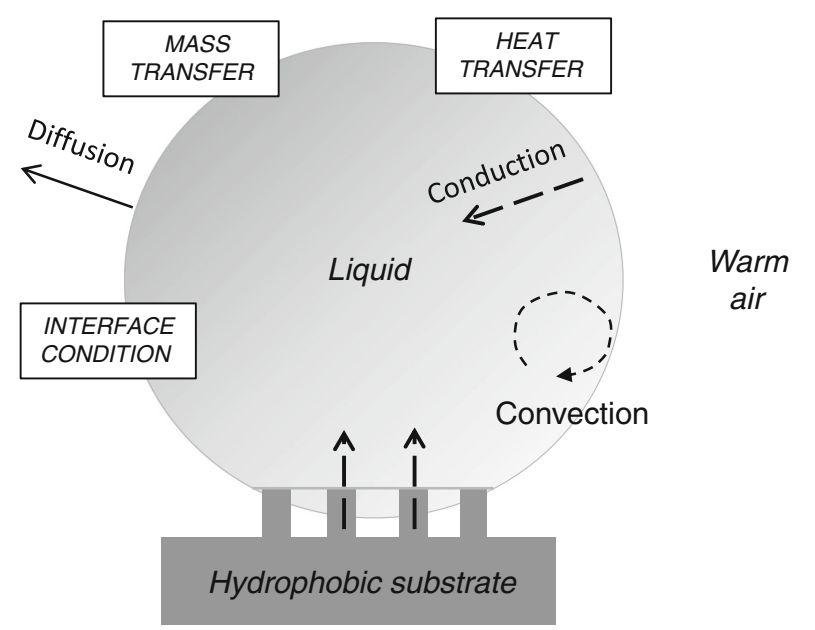

Fig. 2 Diagram of all phenomena involved in the evaporation process for a sessile droplet

law equation according to the vapor concentration and the radial position of the droplet using a spherical surface $\left(A=4 \pi R^{2}\right)$ (Fig. 3) (Maxwell 1890). The rate of mass loss by evaporation is provided by the following:

$$
-\frac{d m}{d t}=-4 \pi R^{2} D_{v} \frac{d c}{d R}
$$

where $m$ is the mass $(\mathrm{kg}), t$ the time (s), $R$ the radial position from the center of the droplet $(\mathrm{m}), D_{v}$ the diffusion coefficient of vapor in air $\left(\mathrm{m}^{2} \cdot \mathrm{s}^{-1}\right)$, and $c$ the vapor concentration $\left(\mathrm{kg} \cdot \mathrm{m}^{-3}\right)$

Taking the vapor concentration at an infinite distance from the droplet center, $c_{\infty}$, and the vapor concentration at the droplet surface, $c_{s}$, Eq. 1 can be integrated between the boundary conditions: $c=c_{s}$ when $R=R_{s}$, and $c=c_{\infty}$ when $R=R_{\infty}$ :

$$
\begin{gathered}
\int_{c_{s}}^{c_{\infty}} d c=\frac{1}{-4 \pi D_{v}} \int_{R_{S}}^{R_{\infty}} \frac{1}{R^{2}} d \mathrm{R} \\
-\frac{d m}{d t}=4 \pi R_{s} D_{v}\left(c_{s}-c_{\infty}\right)
\end{gathered}
$$

where $R_{S}$ is the radius of a spherical droplet (m), $R_{\infty}$ the infinite distance from the center of the droplet $(\mathrm{m}), c_{S}$ the vapor concentration at the droplet surface $\left(\mathrm{kg} \cdot \mathrm{m}^{-3}\right)$, and $c_{\infty}$ the vapor concentration at an infinite distance from the droplet surface $\left(\mathrm{kg} . \mathrm{m}^{-3}\right)$

In Eq. 3, the rate of evaporation is proportional to the radius of the spherical droplet (large droplets evaporate faster than small droplets), but not to the surface area of the droplet, in contrast to measurements performed in a vacuum where the evaporation process is forced.

If vapor obeys the ideal gas law, the vapor concentration can be expressed by the partial pressure of the vapor: 




Fig. 3 Diagram of single-droplet drying configuration corresponding to the model proposed by Maxwell

$$
-\frac{d m}{d t}=4 \pi R_{s} D_{v} \frac{M}{\Re T}\left(P_{S}-P_{\infty}\right)
$$

where $M$ is the molecular weight $\left(\mathrm{g} \cdot \mathrm{mol}^{-1}\right), T$ the temperature $(\mathrm{K}), \mathfrak{R}$ the gas constant $\left(\Re=8.31 \mathrm{~J} . \mathrm{K}^{-1} . \mathrm{mol}^{-1}\right), P_{S}$ the partial pressure at the surface, and $P_{\infty}$ the infinite partial pressure.

Here, according to the mass, the rate of droplet mass loss is a function of the radius, the temperature, the partial pressure, and the transport properties of the air surrounding each droplet.

If the rate of evaporation is expressed according to the radius, i.e., $d m / d t=\rho_{L} 4 \pi R_{S}{ }^{2}$ $d R_{S} / d t$, Eq. 4 becomes

$$
-\frac{d R_{S}^{2}}{d t}=\frac{2 D M}{\rho_{L} \mathfrak{R} T}\left(P_{S}-P_{\infty}\right)
$$

which integrates in

$$
R_{s, 0}^{2}-R^{2}=\frac{2 D M}{\rho_{L} \mathfrak{R} T}\left(P_{S}-P_{\infty}\right) t
$$

where $\rho_{L}$ is the density of the liquid and $R_{s, 0}$ the radius of the spherical droplet at $t=0$.

From Eq. 6, the so-called $d^{2}$ law for evaporation follows the form $R^{2}=R^{2}{ }_{0}-\beta t$ (Law 1982). The evaporation coefficient $\beta$ depends on the thermodynamic properties of the droplet liquid and on conditions of the ambiance of the droplets like temperature or velocity (Frohn and Roth 2000). Using the $d^{2}$ law, Frohn and Roth well demonstrated that droplets from the same liquid but with different sizes have the same evaporation coefficient while uniform droplets in terms of sizes but from different liquids present distinct evaporation coefficients. 
On the other hand, Picknett and Bexon (1977) studied evaporation of a pure liquid droplet deposited on a substrate at a uniform temperature and under diffusion control (Picknett and Bexon 1977). They highlighted three distinct modes of evaporation as follows:

- The constant contact angle mode: the contact angle remains constant and the shape of the droplet remains spherical throughout evaporation, whereas both volume and contact radius decrease (Fig. 4a). This is expected for an ideal system including pure liquid and equilibrium state at the interface, which is often the case of a droplet with a contact angle greater than $90^{\circ}$ (Erbil et al. 2002).

- The constant contact area mode: the contact area between the liquid and the substrate remains constant during evaporation, but the contact angle diminishes (Fig. 4b). This situation is expected for a droplet with an initial contact angle of less than $90^{\circ}$ (Erbil et al. 2002).

- A mix of these two modes: either (i) one mode may switch to the other at any time during evaporation or (ii) both contact angle and contact radius may decrease together. The latter situation often occurs at the end of evaporation of the droplet (Yu et al. 2012). The duration of each mode may be influenced by droplet composition and surface properties (microstructure, thermal conductivity, and chemical heterogeneity). For example, when a spherical droplet is deposited on a flat surface, the evaporation rate is slower compared to a free droplet where no substrate limits the evaporation rate. Indeed, the presence of the surface reduces the space into which vapor can diffuse and thus reduces the evaporation rate. When the substrate is taken into account in Eq. 3, the loss of droplet volume with over time can be expressed as follows:

$$
-\rho_{L}\left(\frac{d V}{d t}\right)=4 \pi R_{S} D\left(c_{S}-c_{\infty}\right) f(\theta)
$$

where $\rho_{L}$ is the density of the droplet liquid $\left(\mathrm{kg} . \mathrm{m}^{-3}\right)$ and $f(\theta)$ the function of the contact angle of the droplet.

Different authors have proposed polynomial expression of $f(\theta)$ (Bourges-Monnier and Shanahan 1995; Picknett and Bexon 1977; Rowan et al. 1995), and this is fully described in Erbil's review (Erbil 2012).

a constant contact area

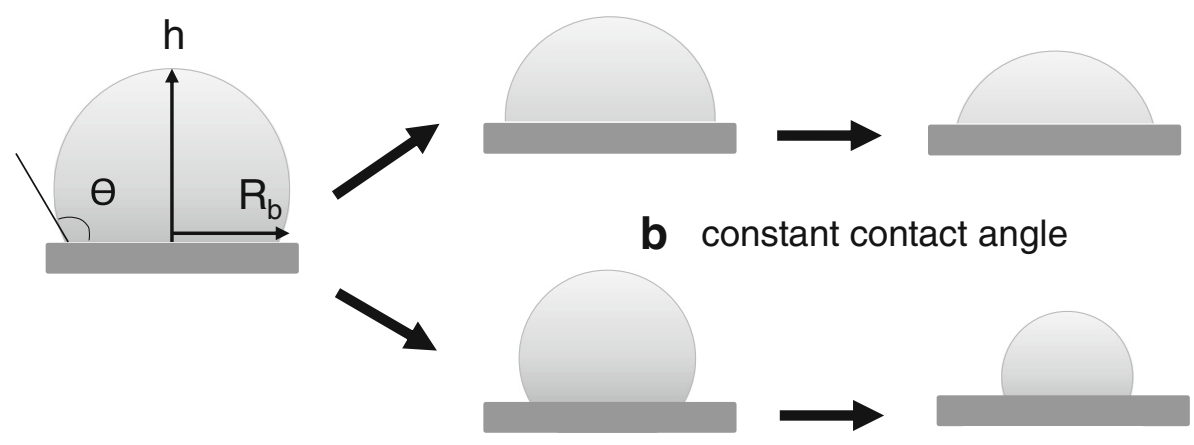

Fig. 4 The two different modes of evaporation for a sessile droplet 


\subsection{Evaporation of single and multicomponent droplet}

In most cases, evaporation takes place for multicomponent droplets with solutes of different physical and chemical properties. Kneer et al. (1993) observed that the temperature and concentration dependence of the liquid properties affected the evaporation process especially with regard to a reduced diffusional resistance (Kneer et al. 1993). Multicomponent droplet evaporation is not trivial with the implication of several phenomena such as unsteady-state kinetics, no-isothermal behavior, internal transport processes, external transport processes, Stefan flow, and physical property variations. Therefore, as mentioned by Frohn and Roth (2000) for a multicomponent droplet, mass and energy transports at the interface and in the interior of the droplet need to be taken into account (Frohn and Roth 2000). Ravindran and Davis (1982) compared theory with experimental results and proposed an expression for diffusion-controlled evaporation rates of two-component droplets (Ravindran and Davis 1982).

Despite the fact that many papers referred to the controlled diffusion approach, this is not the only approach to predict the evaporation rate. In general, the evaporation process can be defined as two simultaneous events at the liquid-air interface: (i) detachment of the water molecule from the interface and (ii) removal of water molecules into the surrounding air by diffusion. The diffusion model usually focuses on event (ii), but other models can be found to describe the process of detachment of molecules during evaporation. They are called kinetics models, transfer rate-limited models, or molecular dynamics models for those focusing on interactions between molecules (Maki and Kumar 2011; Pati et al. 2011). Moreover, many other models can be found in the current literature for more complex evaporation scenarios taking into account convection movement effects (ambient and forced), radiation, heated surface for sessile droplets, the multicomponent droplet aspect, etc. (Ranz and Marshall 1952; Sazhin 2006; Chen and Lin 2005; Zhang and Yang 1983).

\section{Single-droplet studies of particle formation}

This section introduces the fundamental properties of droplet/particle transition, from skin formation to the final shape of the dry particle, properties such as internal flow, sol-gel transition, and the mechanical properties of the solid film. These phenomena occur in a complex system submitted to different gradients: temperature, surface tension, and solute concentration. Investigation of these processes is crucial for the understanding of how they influence the shape of the particle and the physicochemical composition of the skin, which would have an impact on the final powder.

\subsection{Transport mechanisms}

The transport mechanisms of micro- and nanoparticles occurring during evaporation of the droplet have been widely investigated with the sessile droplet method. Competition between various physical mechanisms such as Deegan flow, Marangoni flow, and evaporative flux may occur inside the sessile droplet during evaporation (Fig. 5). Investigations of such flows have been reported in the literature (Majumder et al. 




Fig. 5 Transport mechanisms occurring inside a sessile droplet

2012; Maki and Kumar 2011; Manukyan et al. 2013; Monteux and Lequeux 2011; Zhang et al. 2008).

Deegan flow is a radial outward flow which appears to compensate for the faster evaporation of the thin liquid layer formed at the contact line of the sessile droplet (Deegan et al. 1997). It results in an accumulation of solutes by capillary action from the center to the contact line, forming a solid deposit at the droplet periphery known as a "coffee ring." The ring formation has been reported for a wide range of systems from model suspensions (Hampton et al. 2012) to biological ones (Gorr et al. 2012). Strong modifications of the coffee ring effect have been reported by controlling key parameters such as the contact angle of the droplet (Chen and Evans 2010), the characteristic of the solutes (Zhang et al. 2008), and the nature of the solvents or the drying environment (Majumder et al. 2012; Zhang et al. 2008).

At the air-liquid interface, fluctuations in temperature and solute concentration generate a gradient of surface tension and usually result in Marangoni instability. Convection rolls occur inside the droplet, dragging solutes along the surface to the center. This phenomenon has been widely investigated by experimental and numerical analysis (Ha and Lai 2002; Hu and Larson 2005; MacDonald and Ward 2012; Maki and Kumar 2011). Marangoni in addition to Deegan are two important flows occurring in the droplet evaporation. Marangoni flow is known to counterbalance the Deegan effect by inducing solute circulation towards the droplet center rather than the edge $(\mathrm{Hu}$ and Larson 2006) and leading to a skin formation at the droplet surface (Brutin et al. 2011; Maki and Kumar 2011). Several papers focused on how to facilitate Marangoni flow during droplet evaporation to provide a uniform deposit more desirable for specific applications such as inkjet printing (Sefiane 2014).

The evaporation of water at the droplet surface induces a diffusional flux of solutes from the free surface toward the center. The diffusion coefficients of solutes are estimated using Stokes-Einstein's equation and depend on the internal liquid criteria such as solution viscosity, solute radii, and temperature:

$$
D=\frac{k T}{6 \pi \eta r_{c}}
$$

where $k$ is the Boltzmann's constant $\left(k_{\mathrm{B}}=1.38 \times 10^{-23} \mathrm{~J} . \mathrm{K}^{-1}\right), T$ the temperature $(\mathrm{K}), \eta$ the dynamic viscosity (Pa.s), and $r_{c}$ the component radius (m).

Kim et al. (2003) have estimated different diffusion coefficients of milk components to understand the repartition of solutes inside the particle after droplet drying (Kim et al. 
2003). After the chemical analysis of the surface of the dry particle by using X-ray photoelectron spectroscopy, these authors observed a majority of larger solutes located at the surface such as fat globules and proteins that agree well with the theoretical difference between solute diffusivities.

Finally, two other mechanisms, i.e., the gravitational force and Brownian motion, may also be involved in the droplet evaporation (Fig. 5). Despite the fact that the consequences of these movements are less important than capillary and Marangoni flow ones, these may take place in specific cases.

Gravitational force should be considered when the droplet is large enough to be deformed by gravity, and the solution is a dispersion of solutes which might aggregate during evaporation. Gravity deforms the droplet if its radius is larger than the capillary length:

$$
l_{c}=\sqrt{\frac{\gamma}{g \rho_{L}}}
$$

where $\gamma$ is the surface tension of the liquid-air interface $\left(\mathrm{N} \cdot \mathrm{m}^{-1}\right), g$ the gravitational acceleration $\left(g=9.81 \mathrm{~m} . \mathrm{s}^{-2}\right)$ and $\rho_{L}$ the density of the liquid $\left(\mathrm{kg} . \mathrm{m}^{-3}\right)$.

A few authors focused on the influence of the gravity during the evaporation of a single droplet (Hampton et al. 2012; Sandu and Fleaca 2011; Sommer 2004; Zhu et al. 2010). For droplets large enough (around $1 \mathrm{~mm}$ ), they reported that gravity can contribute to the droplet deformation. Additional to the other flows taking place inside the droplet, gravity can influence the solute segregation and thus the final deposit.

Brownian motion produces random movements of the solute and may influence solute movement inside the droplet. Such random movement results from interactions between the solute and the liquid molecules, driven by the temperature effect. Brownian motion should be taken into account when the droplet liquid is made up of sub-micro solutes. Brownian flow was mentioned by Marín et al. (2011) in the investigation of the solute dynamics during the evaporation of a sessile droplet (Marín et al. 2011). The authors reported different solute layouts according to the flow rate. When the speed is slow, solutes have time to arrange by Brownian motion and form an ordered phase while high-speed solutes are jammed into a disordered phase. However, Brownian motion is often neglected in evaporation system because this latter is widely weaker compared to other transport mechanisms (Rakers et al. 1997; Zhang et al. 2008).

\subsection{Skin formation}

The scientific community has long paid attention to the surface formation process. Investigating the way that the skin of a powder is formed includes two stages, (i) understanding the way that a concentration gradient can be established from the droplet center to the surface and then (ii) the behavior of components that are over-concentrated at the interface. Three main hypotheses originating from theoretical and experimental studies (Kentish et al. 2005; Kim et al. 2003; Wang and Langrish 2009) have been suggested to explain the occurrence of concentration gradients: 
- The first assumption was proposed by Charlesworth and Marshall (1960). They hypothesized that the droplet evaporates in a homogenous way at the beginning of drying due to sufficient transport of water from the interior of the droplet to the surface to maintain saturated conditions (Charlesworth and Marshall 1960). At a critical point, water is lacking at the droplet surface to ensure saturated conditions and a solid phase appears then develops and forms the final skin.

- A second hypothesis was suggested by Meerdink and van't Riet (1995): a water gradient generated by evaporation establishes a component gradient in the opposite direction, from the surface of the droplet to the center. Segregation of components can be explained by different transport velocities depending on the concentration gradients of the components and on the diffusion coefficients of each component (Meerdink and van't Riet 1995). The skin would thus be expected to be composed of higher molecular weight components such as proteins rather than low molecular weight components such as sucrose.

- The last hypothesis was proposed by Fäldt and Bergenståhl (1994). They observed that the more active surface components accumulate at the air-liquid interface and dominate the surface composition of the final powder (Fäldt and Bergenståhl 1994). They therefore assumed that preferential accumulation should occur at the surface with regard to the active surface properties of each component, leading to over-representation of proteins in the composition of the powder skin compared to the bulk composition and to less active surface components (e.g., lactose and fat). These hypotheses are complementary, and all three provide key answers to the way that components are driven to the interface. In addition, the question of skin formation also implies a rheological change of the interface. The nature of the skin is still a matter of debate, it can be defined as a specific region with viscoelastic behavior, a layer of soft gels, or a film of glassy polymers (Okuzono et al. 2006). However, it is accepted that when the volume fraction of components, $\Phi_{i}$, reaches a critical volume fraction, $\Phi_{g}$, a gel or a glassy phase occurs and then three phases coexist at the interface: a gelled skin as a barrier between the liquid core and the surrounding gas. Some studies have proposed that the skin may be promoted by weak diffusion (Maki and Kumar 2011) related to convective evaporation flow, a phenomenon which is usually studied in terms of the Peclet number (Baldwin et al. 2011; Manukyan et al. 2013; Vehring et al. 2007). The Peclet number reflects the extent of convection in relation to diffusion and is commonly expressed by:

$$
P e=\frac{J h_{0}}{D}
$$

where $h_{0}$ is the initial height of the droplet, $J$ the evaporation flux estimated from the loss of volume, $V$, per area unit, $A$, at the beginning of the drying process $J=-(1 / A) d V / d t$, and $D$ is the solute diffusivity (Eq. 8) according to solute size and the viscosity of water.

For a low Peclet number, the diffusion dominates, inducing low concentration gradients at the interfaces, while for a high Peclet number the convection dominates and the vertical gradients of solutes are more pronounced (Maki and Kumar 2011). Okuzono et al. (2006) demonstrated that the formation of the 
skin depends on two parameters, i.e., the initial volume fraction of components, $\Phi_{i}$, and the Peclet number (Fig. 6) (Okuzono et al. 2006). When $\Phi_{i}$ is near $\Phi_{g}$ and $P e$ is high, the skin layer appears easily during drying, whereas no skin is observed for $\Phi_{i}$ much smaller than $\Phi_{g}$ and a low $P e$ value (Ozawa et al. 2006).

\subsection{Mechanical properties of the skin}

When the sol-gel transition is reached at the droplet surface, the skin formed is submitted to considerable capillary stresses induced by the loss of water occurring at the surface. According to the internal properties of the skin, mechanical instability may occur at the surface whatever the drying configuration. For droplets suspended from a capillary, Hassan and Mumford distinguished two kinds of mechanical evolution of the surface (Hassan and Mumford 1996). On the one hand, a smooth, flexible shell is progressively formed during drying: its elasticity allows the particle to undergo inflation or expansion cycles and shrink even after the skin has formed. On the other hand, a more porous and rigid skin crust may be formed, with lower resistance to vapor diffusion. The skin thickens to form a crust, and particles then keep their shape and size until the end of drying. As they cannot expand to resist the internal pressure, fracture formation may occur at the surface resulting in brittle particles.

The final particle shape is governed by the capacity of the skin to deform: for example buckled and collapsed shapes for levitated droplets as reported by Tsapis et al. (2005) who considered that buckling instability is a viscoelastic response of the skin to capillary forces occurring as the water evaporates. The beginning of drying consists of isotropic shrinkage of the droplet, inducing concentration of solutes and increase in surface viscosity. When the skin starts to bulk, these authors hypothesized that the surface would change to an elastic shell.

Identical surface instability may be observed for sessile droplets. Pauchard and Allain (2003) described shape distortions after the sol-gel transition or glassy transition for colloidal and polymer droplets (Pauchard and Allain 2003). They described the occurrence of such mechanical instability and the degree of deformation as a function

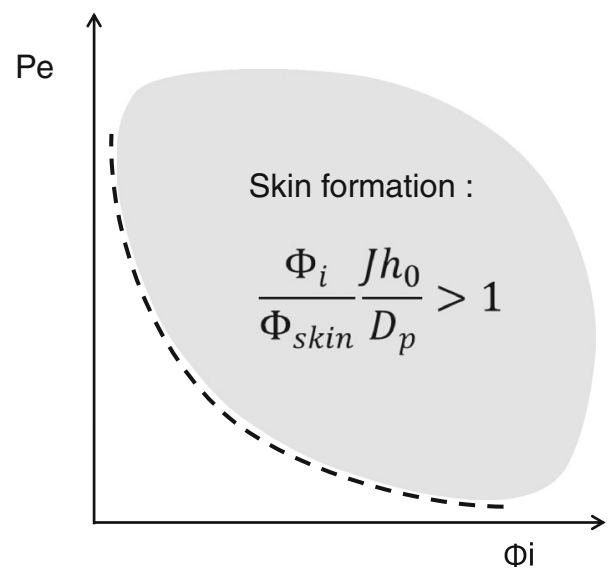

Fig. 6 Skin formation in relation to Peclet number and volume fraction 
of the physicochemical conditions. On the other hand, Sobac and Brutin (2011) identified the nucleation of cracks for a critical concentration of solutes, leading to a typical distribution of fractures around the particle (Sobac and Brutin 2011).

Overall, particle formation seems to be governed by two main factors: the rheological evolution of the material and the occurrence of surface distortions (Pauchard and Allain 2003). These spatial and temporal distortions result from the skin's ability to withstand internal stresses. These mechanical properties of the material thus strongly contribute to the final shape of the particle (Fig. 7).

\section{Single-droplet studies: control of particle features}

The aim of the following section is to provide a brief overview of how experimental conditions can influence particle formation and its physical properties such as the size and shape of particles.

\subsection{Influence of bulk}

Several factors related to the bulk properties have been reported to have strong influences on the quality of the resulting particles.

A pioneering investigation into the impact of the material was undertaken by Walton and Mumford (1999a, b) by means of the drying of a single-droplet system (Walton and Mumford 1999a). In this study, three categories of particle (crystalline, skin forming, and agglomerate) were distinguished according to their drying behaviors and their final

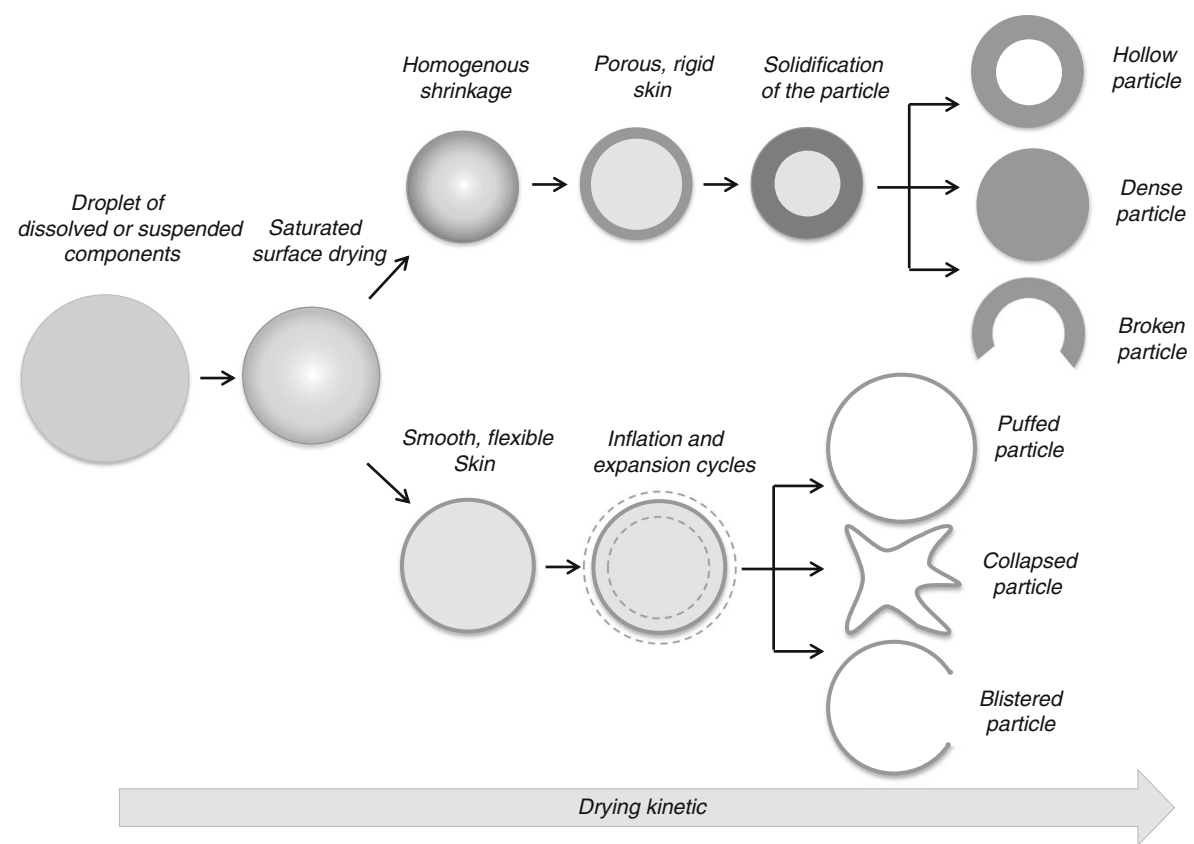

Fig. 7 Diagram showing different ways of particle deformation 
particle shapes. The authors also reported a good agreement between the single particle attributes and those produced by industrial spray drying. In addition, the properties of the solvent have a key role both in determining the drying behavior of the components and in the final structure of particles (Carver and Snyder 2012; Raula et al. 2004; Wan et al. 2013). Moreover, the feed concentration and the viscosity are known to affect particle size. Larger particles are observed for high concentrations, due to the formation of larger droplets induced by a more viscous concentrated solution (Elversson and Millqvist-Fureby 2005; Walton and Mumford 1999b). In addition, the feed concentration and density may also have a strong influence on the shell thickness. A thinner and more flexible shell can be expected for lower concentrations, while a thicker and thus more rigid shell is more likely to be produced with higher feed concentrations (Tsapis et al. 2005). Particles with thicker shells are not able to collapse, as reported by Rogers et al. (2012) for monodispersed droplets and by Fu et al. (2013) for suspended droplets (Rogers et al. 2012; Fu et al. 2013).

The respective intrinsic properties of each component strongly influence the properties of the resulting particles. For example, different crystallization propensities lead to hollow particles in the case of amorphous carbohydrates while porous particles are obtained with crystalline carbohydrates (Elversson and Millqvist-Fureby 2005). The glass transition induces strong rheological modifications which can be manifested through skin formation (Adhikari et al. 2004; Shrestha et al. 2008). Similarly, Werner et al. (2008) related the mechanical stress of the skin at the glass transition temperature to the temperature and the water content of the droplet surface (Werner et al. 2008). Surface activity of components is also known to affect particle shape (Kawakami et al. 2010). Because they are attracted to air-liquid interfaces, surfactants tend to reduce internal movements and modify surface properties. Thus, highly surface-active solutes are likely to induce the formation of spherical and hollow particles (Maa et al. 1997; Paramita et al. 2010).

Also very important are the effects of solubility, molecular weight, and diffusivity of solutes. The importance of solubility on particle size has been reported (Elversson and Millqvist-Fureby 2005; Lin and Gentry 2003). Both studies reported the production of the smallest and most dense particles by the most highly soluble solutes. Moreover, molecular weight has been shown to have a key role in the composition and topography of the particle surface (Kim et al. 2002; Kim et al. 2003). Solutes of high molecular weight have more limited mobility and diffuse more slowly toward the droplet center. Consequently, high molecular weight solutes tend to concentrate at the surface, inducing the earlier formation of a shell at the droplet surface (Fäldt and Bergenståhl 1996). In contrast, minerals are found to be mainly located at the center of the droplet due to their higher diffusivity and their absence of tension-active properties (Gaiani et al. 2006). Finally, the ability of components to bind to water may also affect the drying kinetics and thus influence particle characteristics (Ameri and Maa 2006).

\subsection{Influence of process parameters}

Drying kinetics is of major importance in the particle formation process. Drying kinetics can be managed through various process parameters such as the drying temperature, humidity, droplet size, and feed and air flow rates, all independently.

The influence of the drying temperature in particle formation represents an important focus of research, and many investigations have been carried out (Fang et al. 2012;

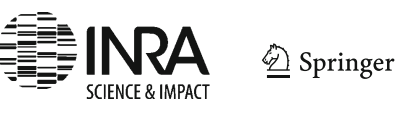


Gaiani et al. 2010; Kim et al. 2009; Maas et al. 2011; Vignolles et al. 2010). It is well known that higher drying temperatures involve faster water evaporation rates at the droplet surface, resulting in rapid component precipitation and crust formation fixing the droplet as soon as it is formed. This has a direct influence on particle size, with the formation of larger particles at higher temperatures and smaller particles at lower temperatures. As for particle shape, the evolution of droplet distortion throughout the drying process was recorded by (Alamilla-Beltrán et al. 2005). The authors reported a greater degree of shrinkage at lower temperatures and inflation and breakage phenomena at higher temperatures for maltodextrin droplets. These temperature particle shape relationships were reported both for suspended droplets (Fu et al. 2013) and for monodispersed droplets (Fang et al. 2012). The strong relationships between the composition of the particle surface and drying temperatures have been widely investigated (Fäldt and Bergenståhl 1996; Kim et al. 2002; Murrieta-Pazos et al. 2012; Nijdam and Langrish 2006). It has been shown that lower temperatures allow the diffusion process to occur in the droplet with a redistribution of components according to their own characteristics (molecular weight, solubility) and affinity (surface activity). In contrast, higher temperatures tend to fix the system through rapid shell formation and maintain the initial component dispersion unchanged. Moreover, according to the heat sensitivity of each component contained in the bulk, a given temperature can induce specific mechanisms such as protein denaturation, leading to the formation of insoluble material (Fang et al. 2012) or crystallization of carbohydrates (Chiou et al. 2008; Maas et al. 2011), all affecting the final surface and shape of a particle.

The way that the droplet is generated represents another key factor. Indeed, droplets of different sizes can be produced according to the characteristics of the spray device, thus affecting their drying kinetics and hence their final particle features (Walzel 2011). For example, a high rotation speed of the rotating wheel and a lower feed concentration result in smaller particles (Littringer et al. 2012b). Smaller particles and faster drying avoid any movement of solids and mostly result in spherical particles. In contrast, atomization of the air flow may be reduced or the nozzle orifice diameter may be increased in order to obtain larger particles (Elversson et al. 2003). As previously described, the formation of the droplet may be strongly influenced by the viscosity, the surface activity of components, and the concentration of solids independently of the spray device.

Finally, the design of the drying system may also affect the drying kinetics. Many investigations have been carried out into interactions between process parameters and particles (Birchal et al. 2005; Chow et al. 2007; Maa et al. 1997; Okuyama and Wuled Lenggoro 2003; Paudel et al. 2013; Vicente et al. 2013). For example, the feed flow rate influences the residence time of a particle in the system and thus governs its drying history, taking into account the relative humidity and the outlet air temperature (Paudel et al. 2013). Increasing the breaking strength of particles has been reported for high feed flow rates (Littringer et al. 2012a). The choice of the type of atomization gas and air flow rate contributes to the formation of specific particle attributes, as reported by Islam and Langrish (2010). These authors observed different crystallization behaviors according to the type of drying gas (Islam and Langrish 2010).

In conclusion, bulk properties and process parameters are intercorrelated fundamental variables in particle attributes as illustrated in Fig. 8. These relationships may be evaluated through the Peclet number and by the diffusivity to evaporation rate ratio, 
mainly governed by the extent of the drying stress. High diffusivity of solutes results in the free migration of components in the droplet, and the surface reaches saturation near the end of drying. This leads to full, solid particles. On the other hand, early surface saturation may be induced by rapid water evaporation from the surface and low diffusivity of solutes. Solutes do not have time to crystallize, and a skin appears instantaneously due to the increase in viscosity at the surface. Different particle shapes may be formed such as hollow, porous particles or collapsed, wrinkled particles according to the mechanical properties of the shell (Vehring 2008; Vicente et al. 2013).

\section{Single-droplet studies of expected functionality of particles}

The above studies showed that both material properties and process parameters are important in determining the drying behavior of the material and the size and shape of particles. Controlling particle features is a major challenge for powder performance in many industrial fields, ranging from food and dairy processing, ceramics, paints, fertilizers, and detergents to the pharmaceutical industry (Paudel et al. 2013). This last section sets out the three main areas currently involved in particle engineering and describes the main applications of these controlled shape particles.

\subsection{Nanotechnology}

In material processing, spray drying allows the production of nanoparticles (1 to $100 \mathrm{~nm}$ ) of metals, semiconductors, and oxides. Spherical silica particles are usually defined by a controllable mesostructure and mesopore size. This represents a

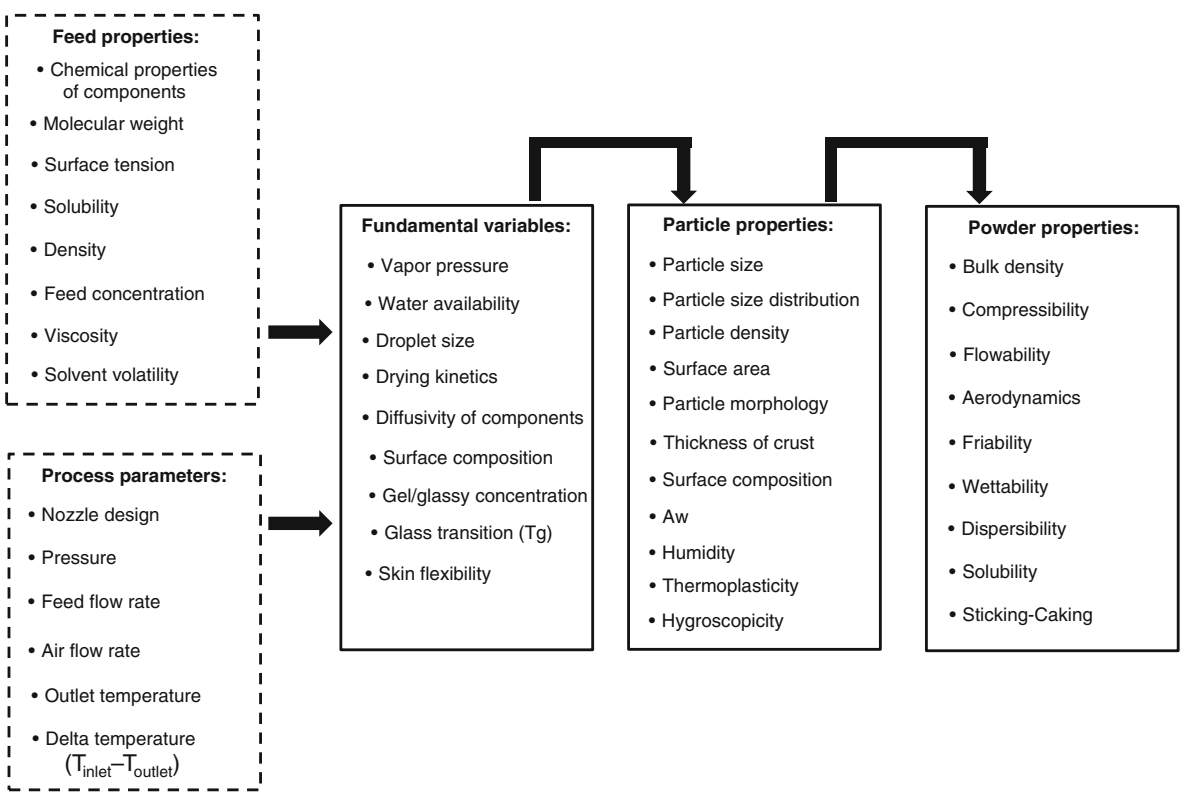

Fig. 8 Diagram of relationships between process parameters, material properties, fundamental variables, and particle and powder properties in spray drying 
fascinating intellectual challenge for potential applications in the production of catalysts, low dielectrically constant chromatography fillers, pigments, microelectronic, and electro-optic elements (Amelia et al. 2012; Iskandar et al. 2007; Okuyama et al. 2006; Okuyama and Wuled Lenggoro 2003).

\subsection{Pharmaceutical applications}

In the pharmaceutical industry, spray drying is used as a particle-tailoring technique for drug delivery. Drug particles consist of a solid pharmaceutical dispersion of active ingredients in an inert carrier matrix (Paudel et al. 2013) such as microspheres for longacting delivery (Ameri and Maa 2006), fine powders for pulmonary delivery (Chow et al. 2007), and vaccine powders for intradermal delivery (Sou et al. 2011). To ensure efficacy of a drug, development has focused on particles designed with specific aerodynamic features such as particle size, density, porosity, surface roughness, and surface energy (Kawakami et al. 2010). With their reduced size and hence their larger specific surface area, nanoparticles make it possible to increase the absorption rate of principle active ingredients, improve their bioavailability, and permit their diffusion to the target (Arpagaus 2012). Dispersibility of inhaled particles is essential for aerosol systems, and this depends on the physical properties of the particles and their potential interactions with others (Raula et al. 2004). For example, corrugated particles with an irregular surface lower the area of contact between particles and limit powder cohesiveness (Chew and Chan 2001) and lower density particles are more likely to travel along airstream lines and reach deep into the lung for effective deposition (Maa et al. 1999). On the other hand, from a manufacturing point of view, compaction and dispersion of powders are very important to ensure successful calibration of tablets and capsules. These properties can be improved by particle size enlargement, increased particle density, or else monitoring the particle morphology.

\subsection{Food industry applications}

Spray drying is widely used in food technology to extend the stability of a product, make its storage easier, and create new ingredients. Research is being carried out on the properties of food powders, including studies to improve their handling and transport during the manufacturing process by controlling the size and density of particles (Barbosa-Cánovas and Juliano 2005; Fitzpatrick and Ahrne 2005; Sharma et al. 2012). Another focus of current development involves the relationships between particle surface composition and powder properties. The influences of surface composition have been established on wall deposition during spray drying (Adhikari et al. 2000; Wang and Langrish 2009), particle agglomeration during storage, and the rehydration ability of powders (Fang et al. 2012). Moreover, in the food industry, there is considerable interest in developing agglomerated, coated, and encapsulated particles by spray drying. The ingredient of interest is totally enveloped by an inert coating material and preserved in the core of the particle. These "smart" particles are used to reduce the reactivity of the "core" with the environment, control the release of the ingredient, mask the core taste, and promote easier handling (Gharsallaoui et al. 2007).

Finally, spray drying techniques offer various factors key to controlling particle formation, thus resulting in a wide range of particles of defined size, shape, surface 
properties, and density and providing aerodynamic characteristics which are valuable for many prospective applications. Powder science and technology are making considerable progress by creating new opportunities to enhance material performance by means of particle engineering.

Spray drying represents a one-step method for producing complex formulations with specific particle characteristics. Spray drying can serve not only as an effective research tool but can also aid a manufacturing on large scale.

\section{Conclusion}

Spray drying is a unit process operation that has been demonstrated to be very valuable in the development of high added value products. Understanding the process of droplet/ particle transition and identifying the key factors governing the final particle features are essential to achieving optimal properties. Current and potential applications of controlled particle properties are discussed in this review that presents the major challenges with regard to spray drying technology and the research and development opportunities involved. However, full understanding of drying phenomena and explanation of particle formation are still issues that are not easy to solve on an industrial scale. This review aims to illustrate that a multi-scale approach to spray drying may provide key elements, with the scaling down to a simple system such as single-droplet drying. The main experiments in the drying of single droplets from sessile droplets to free-flying droplets are reported in this paper. In particular, the single-droplet system has contributed extensively to fundamental and applied understanding of drying (evaporation) phenomena. Finally, investigation of a single droplet may represent a key approach to spray drying in order to improve understanding of the chemical and physical mechanisms involved in particle processing and govern the properties of the final particle.

Acknowledgments The authors thank United Pharmaceuticals for funding this work and for many fruitful discussions.

Conflict of interest Céline Sadek, Pierre Schuck, Yannick Fallourd, Nicolas Pradeau, Cécile Le FlochFouéré and Romain Jeantet declare that they have no competing financial interests.

\section{References}

Alexander K, King CJ (1985) Factors governing surface morphology of spray-dried amorphous substances. Dry Technol 3:321-348

Adhikari B, Howes T, Bhandari BR, Truong V (2000) Experimental studies and kinetics of single drop drying and their relevance in drying of sugar-rich foods: a review. Int Food Prop 3:323-351

Adhikari B, Howes T, Bhandari BR, Troung V (2004) Effect of addition of maltodextrin on drying kinetics and stickiness of sugar and acid-rich foods during convective drying: experiments and modelling. J Food Eng 62:53-68

Alamilla-Beltrán L, Chanona-Pérez JJ, Jiménez-Aparicio AR, Gutiérrez-López GF (2005) Description of morphological changes of particles along spray drying. J Food Eng 67:179-184

Amelia R, Wu WD, Chen XD, Selomulya C (2012) Assembly of magnetic microcomposites from low $\mathrm{pH}$ precursors using a novel micro-fluidic-jet-spray-dryer. Chem Eng Res Des 90:150-157 
Ameri M, Maa YF (2006) Spray drying of biopharmaceuticals: stability and process considerations. Dry Technol 24:763-768

Arpagaus C (2012) A novel laboratory-scale spray dryer to produce nanoparticles. Dry Technol 30:1113-1121

Bahadur J, Sen D, Mazumder S, Paul B, Bhatt H, Singh SG (2011) Control of buckling in colloidal droplets during evaporation-induced assembly of nanoparticles. Langmuir 28:1914-1923

Baldwin KA, Granjard M, Willmer DI, Sefiane K, Fairhurst DJ (2011) Drying and deposition of poly(ethylene oxide) droplets determined by Péclet number. Soft Matter 7819-7826

Barbosa-Cánovas GV, Juliano P (2005) In: Taylor S (ed) Advances in food and nutrition research, vol 49. California, Elsevier, pp 233-307

Birchal VS, Passos ML, Wildhagen GRS, Mujumdar AS (2005) Effect of spray-dryer operating variables on the whole milk powder quality. Dry Technol 23:611-636

Bourges-Monnier C, Shanahan MER (1995) Influence of evaporation on contact angle. Langmuir 11:2820-2829

Brutin D, Sobac B, Loquet B, Sampol J (2011) Pattern formation in drying drops of blood. J Fluid Mech 667: 85-95

Cabazat AM, Guéna G (2010) Evaporation of macroscopic sessile droplets. Soft Matter 6:2591-2612

Carver KM, Snyder RC (2012) Unexpected polymorphism and unique particle morphologies from monodisperse droplet evaporation. Ind Eng Chem Res 51:15720-15728

Charlesworth DH, Marshall WR (1960) Evaporation from drops containing dissolved solids. AIChE J 6:9-23

Chen LF, Evans JRG (2010) Drying of colloidal droplets on superhydrophobic surfaces. J Colloid Interface Sci 351:283-287

Chen XD, Lin SXQ (2005) Air drying of milk droplet under constant and time-dependent conditions. Bioeng Food Nat Prod 51:1790-1799

Chen XL, Boyko V, Rieger J, Reinhold F, Reck B, Perlich J, Gehrke R, Men YF (2012) Buckling-induced structural transition during the drying of a polymeric latex droplet on a solid surface. Soft Matter 8: 12093-12098

Chew N, Chan HK (2001) Use of solid corrugated particles to enhance powder aerosol performance. Pharm Res 18:1570-1577

Chiou D, Langrish TAG, Braham R (2008) The effect of temperature on the crystallinity of lactose powders produced by spray drying. J Food Eng 86:288-293

Chow AHL, Tong HHY, Chattopadhyay P, Shekunov BY (2007) Particle engineering for pulmonary drug delivery. Pharm Res 24:411-437

Davis EJ, Ravindran P, Ray AK (1981) Single aerosol-particle studies. Adv Colloid Interf Sci 15:1-24

Deegan RD, Bakajin O, Dupont TF, Huber G, Nagel SR, Witten TA (1997) Capillary flow as the cause of ring stains from dried liquid drops. Nature 389:827-829

Elsayed TM, Wallack DA, King CJ (1990) Changes in particle morphology during drying of drops of carbohydrate solutions and food liquids. 1. Effects of composition and drying conditions. Ind Eng Chem Res 29:2346-2354

Elversson J, Millqvist-Fureby A (2005) Particle size and density in spray drying - effects of carbohydrate properties. J Pharm Sci 94:2049-2060

Elversson J, Millqvist-Fureby A, Alderborn G, Elofsson U (2003) Droplet and particle size relationship and shell thickness of inhalable lactose particles during spray drying. J Pharm Sci 92:900-910

Erbil HY (2012) Evaporation of pure liquid sessile and spherical suspended drops: a review. Adv Colloid Interf Sci 170:67-86

Erbil HY, McHale G, Newton MI (2002) Drop evaporation on solid surfaces: constant contact angle mode. Langmuir 18:2636-2641

Eslamian M, Ashgriz N (2007) Evaporation and evolution of suspended solution droplets at atmospheric and reduced pressures. Dry Technol 25:999-1010

Fäldt P, Bergenståhl B (1994) The surface composition of spray-dried protein-lactose powders. Colloids Surf A: Physicochem Eng Asp 90:183-190

Fäldt P, Bergenståhl B (1996) Spray-dried whey protein/lactose/soybean oil emulsions. 1. Surface composition and particle structure. Food Hydrocoll 10:421-429

Fang Y, Rogers S, Selomulya C, Chen XD (2012) Functionality of milk protein concentrate: effect of spray drying temperature. Biochem Eng J 62:101-105

Fitzpatrick JJ, Ahrne L (2005) Food powder handling and processing: industry problems, knowledge barriers and research opportunities. Chem Eng Process 44:209-214

Frohn A, Roth N (2000) In: Frohn A, Roth N (eds) Dynamics of droplets. New York, Springer

Fu N, Woo MW, Chen XD (2012) Single droplet drying technique to study drying kinetics measurement and particle functionality: a review. Dry Technol 30:1771-1785 
Fu N, Woo MW, Selomulya C, Chen XD (2013) Shrinkage behaviour of skim milk droplets during air drying. J Food Eng 116:37-44

Gaiani C, Ehrhardt JJ, Scher J, Hardy J, Desobry S, Banon S (2006) Surface composition of dairy powders observed by X-ray photoelectron spectroscopy and effects on their rehydration properties. Colloids Surf B: Biointerfaces 49:71-78

Gaiani C, Morand M, Sanchez C, Tehrany EA, Jacquot M, Schuck P, Jeantet R, Scher J (2010) How surface composition of high milk proteins powders is influenced by spray-drying temperature. Colloids Surf B: Biointerfaces 75:377-384

Gharsallaoui A, Roudaut G, Chambin O, Voilley A, Saurel R (2007) Applications of spray-drying in microencapsulation of food ingredients: an overview. Food Res Int 40:1107-1121

Gorr HM, Zueger JM, Barnard JA (2012) Characteristic size for onset of coffee-ring effect in evaporating lysozyme-water solution droplets. J Phys Chem B 116:12213-12220

Ha VM, Lai CL (2002) Onset of Marangoni instability of a two-component evaporating droplet. Int J Heat Mass Transf 45:5143-5158

Hampton MA, Nguyen TAH, Nguyen AV, Xu ZP, Huang L, Rudolph V (2012) Influence of surface orientation on the organization of nanoparticles in drying nanofluid droplets. J Colloid Interface Sci 377:456-462

Hassan HM, Mumford C (1996) Mechanisms of drying of skin-forming materials; the significance of skin formation and a comparison between three types of material. Dry Technol 14:1763-1777

Hennet L, Cristiglio V, Kozaily J, Pozdnyakova I, Fischer HE, Bytchkov A, Drewitt JWE, Leydier M, Thiaudière D, Gruner S, Brassamin S, Zanghi D, Cuello GJ, Koza M, Magazù S, Greaves GN, Price DL (2011) Aerodynamic levitation and laser heating. Eur Phys J Spec Top 196:151-165

$\mathrm{Hu} \mathrm{H}$, Larson RG (2005) Analysis of the effects of Marangoni stresses on the microflow in an evaporating sessile droplet. Langmuir 21:3972-3980

Hu H, Larson RG (2006) Marangoni effect reverses coffee-ring depositions. J Phys Chem B 110:7090-7094

Iskandar F, Nandiyanto ABD, Yun KM, Hogan CJ, Okuyama K, Biswas P (2007) Enhanced photocatalytic performance of brookite $\mathrm{TiO} 2$ macroporous particles prepared by spray drying with colloidal templating. Adv Mater 19:1408-1412

Islam MIU, Langrish TAG (2010) The effect of different atomizing gases and drying media on the cristallization behavior of spray dried powders. Dry technol 28:1035-1043

Kawakami K, Sumitani C, Yoshihashi Y, Yonemochi E, Terada K (2010) Investigation of the dynamic process during spray-drying to improve aerodynamic performance of inhalation particles. Int J Pharm 390:250259

Kentish S, Davidson M, Hassan H, Bloore C (2005) Milk skin formation during drying. Chem Eng Sci 60: 635-646

Kim H, Cho S (2006) The evaporation of a droplet produced from a liquid surface. J Korean Phys Soc 49: 486-489

Kim EHJ, Chen XD, Pearce D (2002) Surface characterization of four industrial spray-dried dairy powders in relation to chemical composition, structure and wetting property. Colloids Surf B: Biointerfaces 26:197-212

Kim EHJ, Dong Chen X, Pearce D (2003) On the mechanisms of surface formation and the surface compositions of industrial milk powders. Dry Technol 21:265-278

Kim EHJ, Chen XD, Pearce D (2009) Surface composition of industrial spray-dried milk powders. 2. Effects of spray drying conditions on the surface composition. J Food Eng 94:169-181

Kneer R, Schneider M, Noll B, Wittig S (1993) Diffusion controlled evaporation of a multicomponent droplet: theoretical studies on the importance of variable liquid properties. Int J Heat Mass Transf 36:2403-2415

Law CK (1982) Recent advances in droplet vaporization and combustion. Prog Energy Combust Sci 8:171201

Lin SXQ, Chen XD (2002) Improving the glass-filament method for accurate measurement of drying kinetics of liquid droplets. Chem Eng Res Des 80:401-410

Lin JC, Gentry JW (2003) Spray drying drop morphology: experimental study. Aerosol Sci Technol 37:15-32

Littringer EM, Mescher A, Eckhard S, Schrottner H, Langes C, Fries M, Griesser U, Walzel P, Urbanetz NA (2012a) Spray drying of mannitol as a drug carrier-the impact of process parameters on product properties. Dry Technol 30:114-124

Littringer EM, Mescher A, Schroettner H, Achelis L, Walzel P, Urbanetz NA (2012b) Spray dried mannitol carrier particles with tailored surface properties - the influence of carrier surface roughness and shape. Eur J Pharm Biopharm 82:194-204

Maa YF, Costantino HR, Nguyen PA, Hsu CC (1997) The effect of operating and formulation variables on the morphology of spray-dried protein particles. Pharm Dev Technol 2:213-223 
Maa YF, Nguyen PA, Sweeney T, Shire SJ, Hsu CC (1999) Protein inhalation powders: spray drying vs spray freeze drying. Pharm Res 16:249-254

Maas SG, Schaldach G, Littringer EM, Mescher A, Griesser UJ, Braun DE, Walzel PE, Urbanetz NA (2011) The impact of spray drying outlet temperature on the particle morphology of mannitol. Powder Technol 213:27-35

MacDonald BD, Ward CA (2012) Onset of Marangoni convection for evaporating sessile droplets. J Colloid Interface Sci 383:198-207

Majumder M, Rendall CS, Eukel JA, Wang JYL, Behabtu N, Pint CL, Liu TY, Orbaek AW, Mirri F, Nam J, Barron AR, Hauge RH, Schmid HK, Pasquali M (2012) Overcoming the "coffeestain" effect by compositional Marangoni-flow-assisted drop-drying. J Phys Chem B 116:65366542

Maki KL, Kumar S (2011) Fast evaporation of spreading droplets of colloidal suspensions. Langmuir 27: $11347-11363$

Mangel J, Baer E (1962) The evaporation of water drops from a Teflon surface. Chem Eng Sci 17:705-706

Manukyan S, Sauer HM, Roisman IV, Baldwin KA, Fairhurst DJ, Liang H, Venzmer J, Tropea C (2013) Imaging internal flows in a drying sessile polymer dispersion drop using spectral radar optical coherence tomography (SR-OCT). J Colloid Interface Sci 395:287-293

Marín AG, Gelderblom H, Lohse D, Snoeijer JH (2011) Order-to-disorder transition in ring-shaped colloidal stains. Phys Rev Letters 107:085502(1)-085502(4)

Marín AG, Gelderblom H, Susarrey-Arce A, van Houselt A, Lefferts L, Gardeniers JGE, Lohse D, Snoeijer JH (2012) Building microscopic soccer balls with evaporating colloidal fakir drops. Proc Natl Acad Sci 109: $16455-16458$

Maxwell JC (1890) In: Niven WD (ed) Collected scientific papers. Cambridge University Press, Cambridge

McHale G, Aqil S, Shirtcliffe NJ, Newton MI, Erbil HY (2005) Analysis of droplet evaporation on a superhydrophobic surface. Langmuir 21:11053-11060

Meerdink G, van't Riet K (1995) Modelling segregation of solute material during drying of liquid foods. AIChE J 41:732-736

Miura K, Miura T, Ohtani S (1977) Heat and mass transfer to and from droplets. Am Inst Chem Eng Symp Ser 73:95-102

Monteux C, Lequeux F (2011) Packing and sorting colloids at the contact line of a drying drop. Langmuir 27: 2917-2922

Murrieta-Pazos I, Gaiani C, Galet L, Scher J (2012) Composition gradient from surface to core in dairy powders: agglomeration effect. Food Hydrocoll 26:149-158

Nijdam JJ, Langrish TAG (2006) The effect of surface composition on the functional properties of milk powders. J Food Eng 77:919-925

Okuyama K, Wuled Lenggoro I (2003) Preparation of nanoparticles via spray route. Chem Eng Sci 58:537547

Okuyama K, Abdullah M, Lenggoro IW, Iskandar F (2006) Preparation of functional nanostructured particles by spray drying. Adv Powder Technol 17:587-611

Okuzono T, Ozawa K, Doi M (2006) Simple model of skin formation caused by solvent evaporation in polymer solutions. Phys Rev Letters 97:1-4

Ozawa K, Okuzon T, Doi M (2006) Diffusion-process during drying to cause the skin formation in polymer solutions. Jpn J Appl Phys 45:8817-8822

Paramita V, Iida K, Yoshii H, Furuta T (2010) Effect of additives on the morphology of spray-dried powder. Dry Technol 28:323-329

Parisse F, Allain C (1997) Drying of colloidal suspension droplets: experimental study and profile renormalization. Langmuir 13:3598-3602

Pati S, Chakraborty S, Som SK (2011) Influence of ambient vapor concentration on droplet evaporation in a perspective of comparison between diffusion controlled model and kinetic model. Int J Heat Mass Transf $54: 4580-4584$

Pauchard L, Allain C (2003) Mechanical instability induced by complex liquid desiccation. Comptes Rendus Physique 4:231-239

Pauchard L, Couder Y (2004) Invagination during the collapse of an inhomogeneous spheroidal shell. Europhys Letters 66:667-673

Paudel A, Worku ZA, Guns JMS, Guns S, Van den Mooter G (2013) Manufacturing of solid dispersions of poorly water soluble drugs by spray drying: formulation and process considerations. Int $\mathrm{J}$ Pharm 453 : 253-284

Perdana J, Fox M, Schutyser M, Boom R (2013) Mimicking spray drying by drying of single droplets deposited on a flat surface. Food Bioprocess Technol 6:964-977 
Picknett RG, Bexon R (1977) Evaporation of sessile or pendant drops in still air. J Colloid Interface Sci 61: 336-350

Rakers S, Chi LF, Fuchs H (1997) Influence of the evaporation rate on the packing order of polydisperse latex monofilms. Langmuir 13:7121-7124

Ranz WE, Marshall WR (1952) Evaporation from drops. Chem Eng Prog 48:141-146

Raula J, Eerikainen H, Kauppinen EI (2004) Influence of the solvent composition on the aerosol synthesis of pharmaceutical polymer nanoparticles. Int J Pharm 284:13-21

Ravindran P, Davis J (1982) Multicomponent evaporation of single aerosol droplets. J Colloid Interface Sci $85: 278-288$

Rogers S, Wu WD, Lin SXQ, Chen XD (2012) Particle shrinkage and morphology of milk powder made with a monodisperse spray dryer. Biochem Eng J 62:92-100

Rowan SM, Newton MI, McHale G (1995) Evaporation of microdroplets and the wetting of solid-surfaces. J Phys Chem 99:13268-13271

Sakai I, Murai K, Jiang L, Umesaki N, Honma T, Kitano A (2005) Aerodynamic levitation apparatus for structure study of high temperature materials coupled with Debye-Scherrer camera at BL19B2 of SPring8. J Electron Spectrosc Relat Phenom 144-147:1011-1013

Sandu I, Fleaca CT (2011) The influence of gravity on the distribution of the deposit formed onto a substrate by sessile, hanging, and sandwiched hanging drop evaporation. J Colloid Interface Sci 358:621-625

Sazhin SS (2006) Advanced models of fuel droplet heating and evaporation. Prog Energy Combust Sci 32: $162-214$

Schiffter H, Lee G (2007) Single-droplet evaporation kinetics and particle formation in an acoustic levitator. Part 1: evaporation of water microdroplets assessed using boundary-layer and acoustic levitation theories. J Pharm Sci 96:2274-2283

Schutyser M, Perdana J, Boom R (2012) Single droplet drying for optimal spray drying of enzymes and probiotics. Trends Food Sci Technol 27:73-82

Sefiane K (2014) Patterns from drying drops. Adv Colloid Interf Sci 206:372-381

Sharma A, Jana AH, Chavan RS (2012) Functionality of milk powders and milk-based powders for end use applications - a review. Compr Rev Food Sci Food Saf 11:518-528

Shrestha AK, Howes T, Adhikari BP, Bhandari BR (2008) Spray drying of skim milk mixed with milk permeate: effect on drying behavior, physicochemical properties, and storage stability of powder. Dry Technol 26:239-247

Sobac B, Brutin D (2011) Structural and evaporative evolutions in desiccating sessile drops of blood. Phys Rev E 84:1-5

Sommer AP (2004) Limits of the impact of gravity on self-organizing nanospheres. J Phys Chem B 108:8096-8098

Sou T, Meeusen EN, de Veer M, Morton DAV, Kaminskas LM, McIntosh MP (2011) New developments in dry powder pulmonary vaccine delivery. Trends Biotechnol 29:191-198

Tsapis N, Dufresne ER, Sinha SS, Riera CS, Hutchinson JW, Mahadevan L, Weitz DA (2005) Onset of buckling in drying droplets of colloidal suspensions. Phys Rev Lett 94:018302(1)-018302(4)

Vehring R (2008) Pharmaceutical particle engineering via spray drying. Pharm Res 25:999-1022

Vehring R, Foss WR, Lechuga-Ballesteros D (2007) Particle formation in spray drying. J Aerosol Sci 38:728-746

Vicente J, Pinto J, Menezes J, Gaspar F (2013) Fundamental analysis of particle formation in spray drying. Powder Technol 247:1-7

Vignolles ML, Lopez C, Le Floch-Fouere C, Ehrhardt JJ, Mejean S, Jeantet R, Schuck P (2010) Fat supramolecular structure in fat-filled dairy powders: a tool to adjust spray-drying temperatures. Dairy Sci Technol 90:287-300

Walton DE, Mumford CJ (1999a) Spray dried products - characterization of particle morphology. Chem Eng Res Des 77:21-38

Walton DE, Mumford CJ (1999b) The morphology of spray-dried particles - the effect of process variables upon the morphology of spray-dried particles. Chem Eng Res Des 77:442-460

Walzel P (2011) Influence of the spray method on product quality and morphology in spray drying. Chem Eng Technol 34:1039-1048

Wan F, Bohr A, Maltesen MJ, Bjerregaard S, Foged C, Rantanen J, Yang MS (2013) Critical solvent properties affecting the particle formation process and characteristics of celecoxib-loaded PLGA microparticles via spray-drying. Pharm Res 30:1065-1076

Wang S, Langrish T (2009) A review of process simulations and the use of additives in spray drying. Food Res Int 42:13-25

Werner SRL, Edmonds RL, Jones JR, Bronlund JE, Paterson AHJ (2008) Single droplet drying: transition from the effective diffusion model to a modified receding interface model. Powder Technol 179:184-189 
Wu WD, Patel KC, Rogers S, Chen XD (2007) Monodisperse droplet generators as potential atomizers for spray drying technology. Dry Technol 25:1907-1916

Wu WD, Liu WJ, Gengenbach T, Woo MW, Selomulya C, Chen XD, Weeks M (2014) Towards spray drying of high solids dairy liquid: effects of feed solid content on particle structure and functionality. J Food Eng 123:130-135

$\mathrm{Xu}$ W, Choi CH (2012) Effects of surface topography and colloid particles on the evaporation kinetics of sessile droplets on superhydrophobic surfaces. J Heat Transfer 134:051022(1)-051022(7)

Yu YS, Wang ZQ, Zhao YP (2012) Experimental and theoretical investigations of evaporation of sessile water droplet on hydrophobic surfaces. J Colloid Interface Sci 365:254-259

Zhang N, Yang WJ (1983) Evaporation and explosion of liquid drops on a heated surface. Exp Fluids 1:101111

Zhang Y, Yang S, Chen L, Evans JRG (2008) Shape changes during the drying of droplets of suspensions. Langmuir 24:3752-3758

Zhu ZQ, Brutin D, Liu QS, Wang Y, Mourembles A, Xie JC, Tadrist L (2010) Experimental investigation of pendant and sessile drops in microgravity. Microgravit Sci Technol 22:339-345 